\title{
Implementation of vision based 2-DOF underwater Manipulator
}

\author{
Jinpeng Geng ${ }^{1, a}$, Wusheng Chou ${ }^{1}$, Mingjie Dong ${ }^{1}$ \\ ${ }^{1}$ Robotics Institute, Beihang University, Beijing, 100191, P.R. China
}

\begin{abstract}
Manipulator is of vital importance to the remotely operated vehicle (ROV), especially when it works in the nuclear reactor pool. Two degrees of freedom (2-DOF) underwater manipulator is designed to the ROV, which is composed of control cabinet, buoyancy module, propellers, depth gauge, sonar, a monocular camera and other attitude sensors. The manipulator can be used to salvage small parts like bolts and nuts to accelerate the progress of the overhaul. It can move in the vertical direction alone through the control of the second joint, and can grab object using its unique designed gripper. A monocular vision based localization algorithm is applied to help the manipulator work independently and intelligently. Eventually, field experiment is conducted in the swimming pool to verify the effectiveness of the manipulator and the monocular vision based algorithm.
\end{abstract}

Keywords: ROV, 2-DOF manipulator, monocular camera, monocular vision segmentation algorithm

\section{Introduction}

These guidelines, Underwater research and development has becoming faster and faster during the last decade. However, the extreme conditions such as high water pressure, invisibility and non-oxygen become great barriers for human to access directly [1]. Underwater robots are expected and developed as efficient tools for the operation instead of human beings, and ROV is just one of them. Based on this, the manipulator mounted on the ROV is expected to play an important role for complex tasks, such as grabbing small parts like bolts and nuts in the nuclear reactor pool.

There are many manipulators that are developed for industrial purpose but fewer for underwater utility on account of the high sealing requirement in the highpressure underwater environment and complex control method. A visual servoing control system was proposed for a two-link electrically actuated underwater manipulator by Maruthupandi A etc, and its effectiveness had been verified in a laboratory water tank using visual feedback to process the position and tracking control of the 2-link underwater robot manipulator [2]. CC Chang et al. [3] had designed and implemented an intelligent underwater robotic manipulator system, which consisted of a manipulator, ad distance measurement system, water-proof camera, computer, interface technology and task planning technology etc, to enhance the capability of the robotic manipulator to fulfill the need of less-skilled or even non-skilled operators. It has five rotary joints, including waist, shoulder, and elbow, wrist-pitch, and wrist-roll motion, and can accomplish an object grab under water with an ROV system. The 2-DOF manipulator designed and manufactured by us is mounted on an ROV that is used to inspect the reactor pressure vessel and other water-filled infrastructure in the nuclear reactor pool. It has two joints, and can move in the vertical direction alone through the control of the second joint and grab object using its unique designed gripper. A monocular vision based localization algorithm is applied to help the manipulator grab object more independently and intelligently.

The paper is organized as follows. Section 1 presents the underwater robot platform, namely the designed ROV for the nuclear power plant pool. Section 2 describes the 2-DOF manipulator in detail. The monocular vision based localization algorithm and experiments are displayed in Section 3 and Section 4, respectively. Conclusions are drawn in Section 5.

\section{Underwater Robot Platform}

Use The designed and manufactured underwater robot prototype is shown in Figure 1. The robot's weight is 9 kilograms and its size leaving out the 2-DOF manipulator is $370 \mathrm{~mm} \times 312 \mathrm{~mm} \times 340 \mathrm{~mm}$ (length $\times$ width $\times$ height). The ROV is composed of control cabinet, buoyancy module, four propellers, one monocular camera, a depth

\footnotetext{
$\overline{\text { a Jinpeng Geng:buaaalan@126.com }}$
} 
gauge and one 2-DOF manipulator. It is designed to be neutral buoyancy and can stay at any depth under the water. The buoyancy module is made of metal and is hollow to provide the buoyancy and balance the weight of the ROV. The control cabinet with the whole control system inside is made of aluminum alloys to provide sufficient strength and resist to the acidic corrosion of the reactor pool in the nuclear power plant. The ROV is driven by the four propellers with two in the horizontal direction and two in the vertical direction. The designed 2-DOF manipulator is installed in the front below the control cabinet, and is used to salvage small parts like bolts and nuts in the reactor pool with the help of the monocular camera above the manipulator. Besides, the ROV is equipped with many sensors, such as depth gauge, three-axis accelerometer, three-axis gyroscope and threeaxis magnetometer, which are used to get the position and attitude of the ROV.

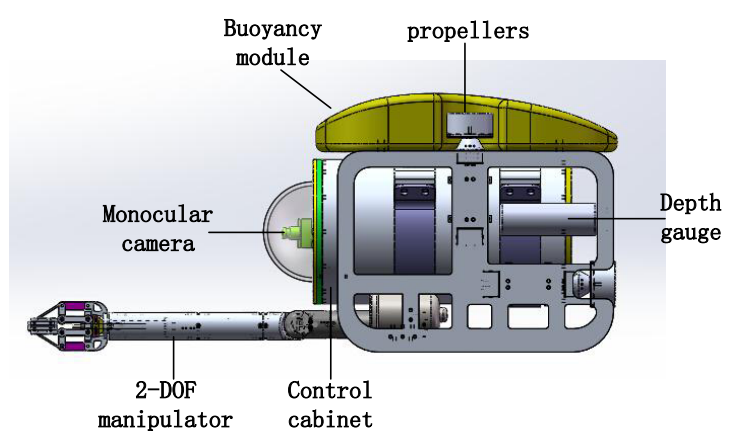

Figure 1. Prototype of the underwater robot

\section{2-DOF Manipulator}

Figures and tables, as originals of good quality and well contrasted, are to be in their final form, ready for reproduction, pasted in the appropriate place in the text. Try to ensure that the size of the text in your figures is approximately the same size as the main text (10 point). Try to ensure that lines are no thinner than 0.25 point.

Figure 1. Caption of the Figure 1. Below the figure.

\subsection{Design of the Joint Transmission Parts}

The joint transmission parts are designed as Figure 2.

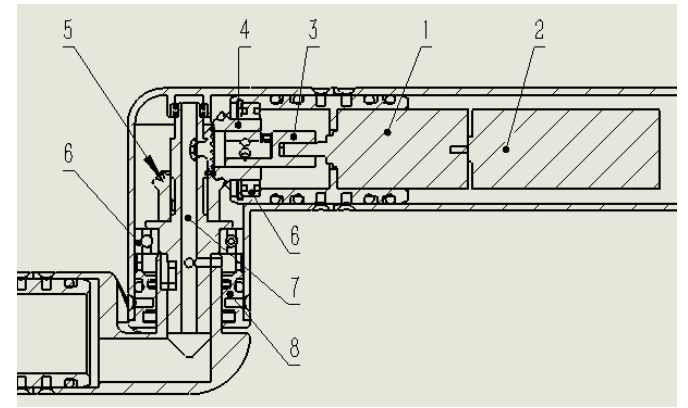

Figure 2. Design of the joint transmission parts (1-planetary reducer; 2-DC motor; 3-coupling; 4,5-bevel gear; 6-deep groove ball bearing; 7-drive shaft; 8 -joint end cover)

The 2-DOF manipulator is driven by the DC motor. The output of the DC motor is delivered to the second joint through planetary reducer and two bevel gears. The interior of the bevel gear and the drive shaft is used to wiring. The DC motors in the first joint and the second joint work alone and have no effect on each other.[4]

\subsection{Design of the gripper transmission parts}

The design of the gripper transmission parts are as Figure 3.

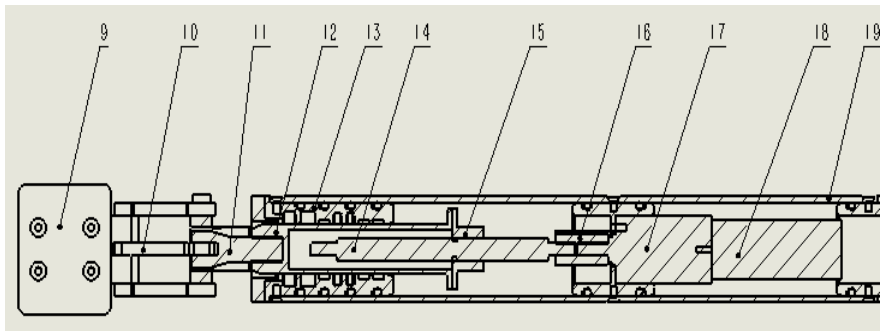

Figure 3. Design of the gripper transmission parts

(9-grip fingers; 10-parallelogram gripper mechanism; 11 gripper driven slider; 12-rod sleeve; 13-end caps; 14-ball screw; 15-ball bearing slide; 16-coupling; 17-DC motor; 18-plantary reducer)

The ball screw is driven by the DC motor through coupling, and then promotes the movement of the parallelogram gripper mechanism and the grip fingers. All the joints have been done the double seal processing to protect the inside parts from water leakage.[5]

The performance parameters of the designed 2-DOF manipulator are as follows,

Working depth: $>\mathbf{5 0} \mathbf{~ m}$

Weight in water: $<2 \mathrm{Kg}$

Bearing capacity: $>\mathbf{0 . 5} \mathrm{Kg}$

Maximum grabbing diameter: $60 \mathrm{~mm}$

The outlook of the entire 2-DOF manipulator is as Figure 4.

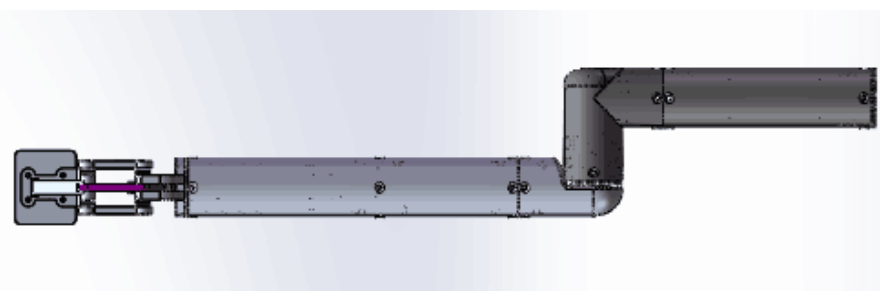

Figure 4. 2-DOF manipulator

Place the figure as close as possible after the point where it is first referenced in the text. If there is a large number of figures and tables it might be necessary to place some before their text citation. If a figure or table is too large to fit into one column, it can be centred across both columns at the top or the bottom of the page. 


\section{Monocular vision based segmentation algorithm}

\subsection{Underwater vision system}

The $\mathrm{R} / \mathrm{C}$ vision system contains a micro 2 -DOF cloud terrace and a CMOS camera. The basic information is as Table 1.

Table 1. Basic information of $\mathrm{R} / \mathrm{C}$ vision system

\begin{tabular}{|c|c|}
\hline Camera & $\begin{array}{c}\text { 600TVL FPV TUNED CMOS } \\
\text { CAMERA }\end{array}$ \\
\hline Imager & $\begin{array}{c}1 / 3 \text { " CMOS;FPV tuned } \\
\text { handling; PAL or NTSC }\end{array}$ \\
\hline Lens & $\begin{array}{c}3.6 \mathrm{~mm} \text { IR coated; } 90^{\circ} \text { diagonal } \\
\text { FOV }\end{array}$ \\
\hline Operation & -20 to $70 \mathrm{C}$ \\
\hline
\end{tabular}

\subsection{Algorithm principle}

Recognizing and separating the target from images are the basis of the monocular vision based localization algorithm. In this paper, a method of target segmentation and extraction is proposed, which includes: The initial separation based on HSI model and the target extraction based on RGB model. Based on the specific needs of the subject, the object is a red ball of a specific size and shape.[7]

Color model can be divided into several categories, such as RGB, YUV, HIS and CIELAB. Different color models have different characteristics and application fields, and no color model can meet all the requirements of users [8]. This paper proposes a method which uses two different color models (RGB and HSI).This method can take advantages of two color models, so as to get higher accuracy of image segmentation results.

RGB color model is shown in Figure 5 is built in a cube 3-D space coordinate system. Three axes representing the red, green, blue, the origin represents black and the farthest distance from the origin point on behalf of white.

HSI color model is shown in Figure 6, its three components constitute a 3-D space double pyramid structure. The HSI model is based on two important facts the first is that I component has nothing to do with the image color information. [10]And the second is that $\mathrm{H}$ and $\mathrm{S}$ components are closely related to way people feel the color. These features make the HSI model suitable for color feature detection and analysis.

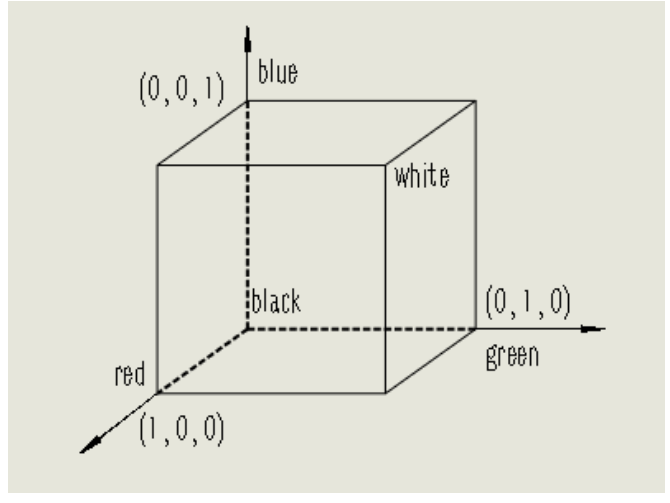

Figure 5 RGB color model

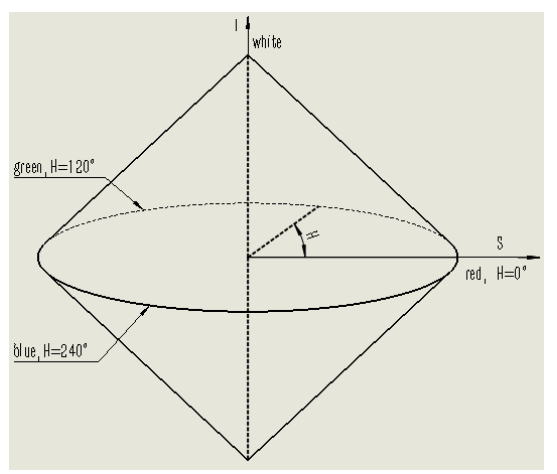

Figure 6 HSI color model

Integrated the characteristics of RGB and HSI model, the algorithm implementation steps are as follows:

(1) Traverse the image, classify the target and background according to the hue $(\mathrm{H})$, saturation $(\mathrm{S})$ and Intensity (I);

(2) Transform the HSI to the RGB model;

(3) Conduct color image segmentation to the RGB model to obtain the final result.

The flow chart is as Figure 7:

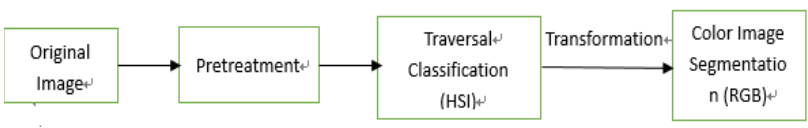

Figure 7 The flow chart of the Algorithm

It's confirmed through a lot of experiment that in order to separate the red ball from background, the range of hue should be $0^{\circ}<\mathrm{H}<45^{\circ}$, saturation should be $S \geq 0.25$ and without any restriction to intensity(I)[11]. The formulas for converting from HSI to RGB are as follow:

$$
\begin{aligned}
& \mathrm{R}=\mathrm{I}(1-\mathrm{S}) \\
& \mathrm{G}=\mathrm{I}\left[1+\frac{s \cos H}{\cos (60-H)}\right] \\
& \mathrm{B}=3 \mathrm{I}-(\mathrm{R}+\mathrm{G})
\end{aligned}
$$

By changing the threshold of $\mathrm{R}, \mathrm{G}$ and $\mathrm{B}$, the color image segmentation is carried out. The concrete range is that $200<\mathrm{R}<260, \quad 70<\mathrm{G}<220$ and $85<\mathrm{B}<230$. The MATLAB simulation results of 
this method as shown in Figure 8, and the results without HSI segmentation as shown in Figure 9.[12]

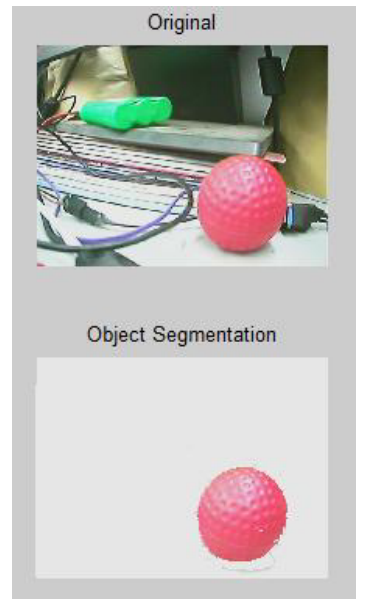

Figure 8 Results of this method segmentation

\section{Field experiment}

Experiments have been done to verify the performance of the designed 2-DOF manipulator, especially its monocular vision based localization algorithm. The waterproof of the manipulator is designed to be IPX-8. And the target of the grasp experiment based on the monocular vision is a red ball under the water. Result of the experiment is as shown in Figure 10.

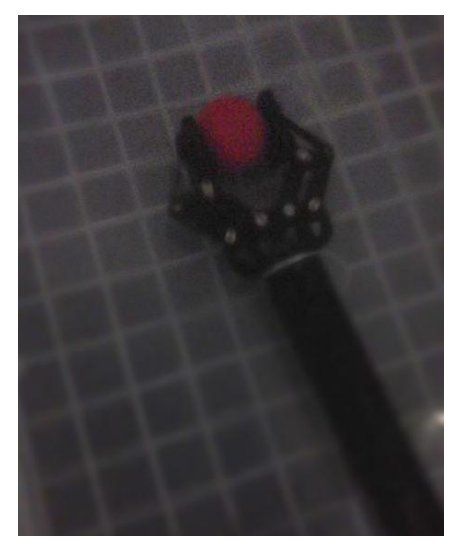

Figure 10. Experiment of grasping performance

The experiment lasted for about one hour, and the designed manipulator still worked normally and showed good waterproof performance. Grasp experiment is a little more difficult because of the exponential attenuation of light and the absorption property of water. The red ball was grasped after a few times, and the automatic fetching test need to be improved in future, and that is our next work.

\section{Conclusions}

The designed manipulator has good waterproof performance and can grasp target. The monocular vision based algorithm also worked.

\section{Acknowledgements}

The authors are grateful for the support of the National Key Basic Research Program of China (973 Project) under grant number 2013CB035503.

\section{References}

1. Ishitsuka M, Ishii K. Development of an underwater manipulator mounted for an AUV [J]. Proceedings of Oceans Mts/ieee, 2005:1811-1816 Vol. 2.

2. Maruthupandi A, Pandian S R. Visual servoing of a 2-link underwater robot manipulator[C]//Underwater Technology (UT), 2015 IEEE. IEEE, 2015: 1-2.

3. Chang $\mathrm{C} \mathrm{C}$, Wang $\mathrm{J} \mathrm{H}$, Lin $\mathrm{C} \mathrm{C}$, et al. The study of remotely teleoperated robotic manipulator system for underwater construction[C]// Underwater Technology, 2004. UT '04. 2004 International Symposium on2004:269 - 276.

4. ChenXin. Study on the dynamics of manipulator. 2007:16-18

5. Scheinman V D. Design of a computer controlled manipulator[R]. Stanford Univ Calif Dept of Computer Science, 1969.

6. Xu Xiaolong, Xu Guohua Vision-based Localization of Outland1000 ROV.2009

7. Aider O A, Hoppenot P, Colle E. A model-based method for indoor mobile robot localization using monocular vision and straight-line correspondences $[\mathrm{J}]$. Robotics and Autonomous Systems, 2005, 52(2): 229-246.

8. Andreas K, Mongi A. Digital Color Image Processing.2010:52-54

9. LiXuan. Research on the Technologies of Underwater Image Segmentation and Object Locatio based on Monocular Vision.2013:88-89

10. Gonzalez R C. Digital image processing[M]. Pearson Education India, 2009.

11. Fairchild M D, Pirrotta E. Predicting the lightness of chromatic object colors using HSI[J]. Color Research \& Application, 1991, 16(6): 385-393.

12. Royer E, Lhuillier M, Dhome M, et al. Monocular vision for mobile robot localization and autonomous navigation[J]. International Journal of Computer Vision, 2007, 74(3): 237-260. 